\title{
Editorial
}

\section{Genetic investigation and counselling of families with hypertrophic cardiomyopathy}

In 1958 Teare described the gross appearances of the heart in eight young people dying suddenly of what became known as hypertrophic cardiomyopathy. ${ }^{1}$ The familial nature of the disease became clear when Teare found himself carrying out a necropsy on a brother of a case in the series. By coincidence on the same day a younger sister attended the Hammersmith Hospital with the clinical signs of hypertrophic cardiomyopathy. Just over 35 years later we are close to understanding the pathogenesis of the disease at the molecular level.

Linkage is the classic method of determining the chromosome on which any abnormal gene is situated. This method is better suited to investigating diseases caused by a single gene rather than by several genes. Coinheritance of the disease with another gene or chromosomal marker at a known position is compared in affected and unaffected members in two or three generations of a family. Such linkage studies showed a gene causing hypertrophic cardiomyopathy on chromosome 14 in about half of the families in which a member had hypertrophic cardiomyopathy. ${ }^{2}$ This gene, now known as cardiomyopathy hypertrophic gene 1 (CMH1), encodes for heavy chain myosin, a component of the myofibrillary apparatus of every myocardial muscle cell. Patients with CMH1-related hypertrophic cardiomyopathy are heterozygous, with one gene encoding for normal myosin and one encoding for the abnormal mutant myosin. Homozygous states are not known, implying that a double dose of the abnormal gene is fatal in utero. This is not quite certain, however, given that both parents would have to be heterozygous and the gene is not common.

Thirty four separate point mutations-each the result of a single amino acid substitution-in the gene code for heavy chain myosin have now been reported. ${ }^{3}$ Most of these so-called missense mutations encode for the head region of the myosin molecule. Occasional families have deletion or rearrangement of a segment of the gene. Such mutations could be expressed as abnormal phenotypes if the abnormal myosin interferes with myofibrillary alignment within the myocyte. This would lead to a misshapen cell and abnormalities of cell to cell alignment. The "disarray" that is such a striking histological feature of hypertrophic cardiomyopathy ${ }^{45}$ is caused by this malalignment and disorganisation.

Current knowledge about the genetics of hypertrophic cardiomyopathy is limited in two important respects. First, if genetic abnormalities of heavy chain myosin account for $50 \%$ of the families with the disease what genes are responsible for the other $50 \%$ ? Linkage studies have shown that abnormalities of at least four other separate genes, all on different chromosomes, can produce a similar clinical picture. ${ }^{6-8}$ These are $\mathrm{CMH} 2$ (chromosome 1), $\mathrm{CMH} 3$ (chromosome 15), and CMH4 (chromosome 11). A fifth gene is as yet unplaced on any chromosome. The proteins encoded by genes CMH 2-5 are not yet known. Thus there are at least five separate genetic forms of hypertrophic cardiomyopathy each with numerous different point mutations, deletions, or abnor- mal registrations of that particular gene. The clinical expressions of each gene form are similar in that hypertrophy without apparent cause is produced; however, the severity of the disease in individuals is variable. It is probably never going to be possible on purely clinical grounds to predict which of the five genes is involved. Once the proteins for which genes CMH 2-5 code are known the concept of myofibrillary dysgenesis may be strengthened or disproved.

The second problem is the range of phenotypic expression that occurs within families-that is, between individuals who have the same mutation of the same gene. There is variability both in the clinical symptoms produced and in the morphology of the heart as shown by echocardiography or at necropsy. Within individual families expression can range from massive septal hypertrophy with chest pain and cardiac failure, through mildly symptomatic disease with obvious echocardiographic appearances of left ventricular hypertrophy, to asymptomatic disease with minimal echocardiographic or electrocardiographic abnormalities. A consequence of subclinical expression is that only formal genetic analysis can identify with certainty all the family members carrying the gene. Sudden death is associated with all degrees of severity. ${ }^{910}$ Thus even family members with asymptomatic disease are at risk. Some of the 34 specific mutations of the myosin heavy chain gene described so far carry a higher risk of sudden death than other mutations. ${ }^{2311}$ Replacement of glutamine at position 403 of the gene by arginine and replacement of tryptophan by arginine at position 719 are both associated with a high frequency of symptoms, including sudden death, and a mean survival of 38 years. The outcome with other substitutions such as leucine for valine at position 908 is more benign. In part the variability depends on the degree to which the amino acid substitution changes the overall charge of the beta heavy chain molecule. ${ }^{11}$ An amino acid may be positive, negative, or neutral and replacement of a charged amino acid by an amino acid of the opposite charge will alter overall charge on the molecule. In addition the position of the mutation may also be important. When valine replaces methionine at position 606 the charge does not change, yet $50 \%$ of individuals with this mutation are dead by $50 .{ }^{11}$ As the catalogue of mutations in the $\beta$ heavy chain myosin grows and the severity of clinical disease in each becomes known it will become possible to assess the prognostic implications for other families with the same mutation. Such data will be of great use in advising other families with the same mutation at the same site. Some of the non-myosin genes also produce less severe disease, for example CMH 3 seems to produce less ventricular hypertrophy and many carriers of the gene are apparently normal. ${ }^{7}$

In part therefore the variability in the clinical expression of hypertrophic cardiomyopathy between families depends on the gene involved and on the nature of the mutation in that gene. This cannot explain the variability in members of the same family with the same gene muta- 
tion. The disease often becomes manifest during the adolescent growth phase and thereafter may remain static or progress. Additional stimuli for left ventricular hypertrophy such as hypertension or undertaking vigorous sport may accentuate the disease but they account for only part of the intrafamilial variability. Perhaps there are other genes that control hypertrophy, and polymorphisms of these genes explain the remaining variability.

There is a mechanism for angiotensin II to affect cardiac myocytes. An insertion/deletion polymorphism of the angiotensin converting enzyme (ACE) gene results in three possible genotypes-these are II, ID, and DD. The DD genotype is associated with increased plasma concentrations of angiotensin converting enzyme. Studies of patients with hypertrophic cardiomyopathy show an excess of patients with the DD genotype. The DD phenotype was more strongly associated with families with a proven risk of sudden death ${ }^{12}$ but these data need confirmation.

Other mechanisms for the variability of phenotypic expression are emerging. $\beta$ Heavy chain myosin can be detected in skeletal muscle although the clinical signs of a skeletal muscle disorder are very rare and unimportant. ${ }^{13}$ In skeletal muscle the ratio of mutant to normal heavy chain myosin varies from patient to patient and some have no mutant myosin in skeletal muscle. Variability of phenotypic expression in the heart may depend on the ratio of normal to mutant myosin in the myocardium. ${ }^{14}$

Where does this explosion of genetic information leave the practising physician who is faced with the problem of advising the family of a living patient with symptomatic hypertrophic cardiomyopathy or relatives of a patient who has died suddenly of the disease? Few cases of hypertrophic cardiomyopathy arise as new mutations. Most therefore will have relatives with the gene. Should these other family members be identified by routine electrocardiograms and echocardiograms or, with more certainty, by genetic screening? There could be conflict between doctors functioning as scientists and as physicians. Scientific knowledge of the disease will advance only if all the members of more families with the disease are studied. The other mutations and genes causing hypertrophic cardiomyopathy need to be identified and then characterised. We need to know what affects gene expression. Some mutations in the myosin molecule are known to increase the risk of sudden death. Prediction of risk can be improved only by further molecular and genetic studies of other affected families. Soon we will classify hypertrophic cardiomyopathy according to the gene responsible and by the exact mutation, and such knowledge will allow the risk of dying from hypertrophic cardiomyopathy to be assessed for each family.

But will identification of an abnormal myosin gene by screening the families of known cases of hypertrophic cardiomyopathy benefit asymptomatic individuals? It may not. Those who are told, after such family screening, that they have an abnormal gene will be anxious and at a disadvantage when applying for life insurance, despite the fact that they have a good chance of living to a ripe old age.

Carriers of the CMH1 gene for heavy chain myosin can be detected by a simple test of DNA extracted from blood lymphocytes. ${ }^{15}$ It is not yet possible to screen families for CMH2-5 but the rate of progress in molecular technology is so great that it is likely that screening for all the genes will soon be possible. Thus even now about half the families with hypertrophic cardiomyopathy can be given accurate genetic information. The arguments for and against knowing who in a particular family has the gene centre on whether the magnitude of the risk of sud- den death or severe clinical symptoms for that individual can be assessed and whether there is any treatment. It is wise to advise gene carriers not to take up competitive sport. Any additional stimulus to cardiac hypertrophy is unlikely to be beneficial, and many with the disease suddenly die during exercise. Hypertrophic cardiomyopathy is a major, although by no means the only, cause of sudden death in trained athletes.

Should anything more active be done particularly in families where a high risk is already declared by other deaths? Workers in centres where many cases are seen have emphasised that it is possible to define the potential mechanisms for the induction of arrhythmias and to give appropriate treatment. ${ }^{16}{ }^{17}$ Sudden death in hypertrophic cardiomyopathy has many different mechanisms ${ }^{17-19}$ but about one third of those with the disease in families with their risk of sudden death already declared have a potentially preventable mechanism capable of initiating an arrhythmia. These include paroxysmal atrial fibrillation, which can be prevented by amiodorone, and left ventricular gradients, which can be treated with $\beta$ blockers or calcium antagonists. Those with sustained refractory arrhythmia may need an implantable defibrillator. ${ }^{3}$ There are algorithms to identify such high risk patients. ${ }^{17-19}$ None the less in most asymptomatic patients, particularly from families where there have been no sudden deaths, no treatable factor that could cause death will be found. Such patients should simply be advised to avoid further stimuli for cardiac hypertrophy such as competitive sport or strenuous physical training.

A controlled randomised trial with the end point of sudden death would be impossible to set up. Families would have to be matched for the gene and the same mutation and the study would last many years. We are likely to have to depend on observational data, at least initially derived from high risk families, for assessing therapy. Thus for some time there will be both strong supporters of screening and risk stratification of those with the gene and those who are more sceptical. ${ }^{20}$ Screening implies at the least advice on life style if not therapy of one type or another. It is, however, naive to believe that we can return to an age of innocence where a physician can deny any knowledge of how a disease may affect a family and how the disease is inherited. Families in which a confirmed case of hypertrophic cardiomyopathy has occurred must be offered screening, must be told of its limitations, and must be counselled about the results. Members of families in which there has been at least one sudden death from hypertrophic cardiomyopathy are more likely to take up screening. In these families uncertainty over who has the disease may cause greater anxiety than the restriction of activities in the family members who carry the gene.

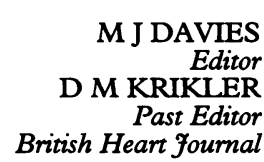

1 Teare D. Asymmetrical hypertrophy of the heart in young adults. $\mathrm{Br} \mathrm{Hear}$ $\exists 1958 ; 20: 1-8$.

2 Watkins $\mathrm{H}$, Rosenzweig A, Hwang D, et al. Characteristics and prognostic implications of myosin missense mutations in familial hypertrophy cardiomyopathy. N Engl f Med 1992;326:1108-14.

3 Roberts R. Molecular genetics-therapy or terror? Circulation 1994;89: 499-504.

4 Maron B, Anan T, Roberts W. Quantitative analysis of the distribution of cardiac muscle cell disorganisation in the left ventricular wall of patients with hypertrophic cardiomyopathy. Circulation 1981;63:882-94.

5 Davies M. The current status of myocardial disarray in hypertrophic cardiomyopathy. Br Heart $₹ 1984 ; 51: 361-3$.

6 Watkins H, MacRae C, Thierfelder L. A disease locus for familial hypertrophic cardiomyopathy maps to chromosome 1q3. Nature Genetics 1993;3:333-7. 
7 Thierfelder L, MacRae C, Watkins H. A familial hypertrophic cardiomyopathy locus maps to chromosome 15q2. Proc Natl Acad Sci USA 1993;90:6270-4.

8 Carrier L, Hengstenberg C, Beckmann J. Mapping of a novel gene for familial hypertrophic cardiomyopathy to chromosome 11. Nature Genetics 1993;4:311-3.

9 Maron B, Roberts W, Epstein S. Sudden death in hypertrophic cardiomyopathy a profile of 78 patients. Circulation 1982;65:1388-94.

10 McKenna W, Stewart J, Niyannopoulos P, McGinty F, Davies M. Hypertrophic cardiomyopathy without hypertrophy: Two families with Hypertrophic cardiomyopathy without hypertrophy: Two families with Heart $\mathcal{f} 1990 ; 63: 287-90$

11 Fananapazir L, Epstein ND. Genotype-phenotype correlations in hypertrophic cardiomyopathy. Comparison of kindreds with distinct and identical $\beta$ myosin heavy chain mutations. Circulation 1994;89:22-31.

12 Marian A, Yu Q-T, Workman R, Greve G, Roberts R. Angiotensinconverting enzyme polymorphism in hypertrophic cardiomyopathy and sudden cardiac death. Lancet 1993;342:1085-6.

13 Yu Q-T, Ifegwu J, Marlan A, et al. Hypertrophic cardiomyopathy mutation is expressed in messenger RNA in skeletal as well as cardiac muscle. Circulation 1993;87:406-12.

14 Fananapazir L, Cuda G, Sellers J, Epstein N. Differences in motility activity of $\beta$ myosin from skeletal muscle in hypertrophic cardiomyopathy kindreds with independent $\beta$-myosin heavy chain gene mutation resulting in the identical 741 gly-arg amino acid substitution [abstr] Circulation 1993;88:1-343.

15 Rosenzweig A, Watkins H, Hwang D, et al. Preclinical diagnosis of familial hypertrophic cardiomyopathy by genetic analysis of blood lymphocytes. N Engl f Med 1991;325:1753-60.

16 McKenna W, Oakley C, Krikler D, Goodwin J. Improved survival with amiodarone in patients with hypertrophic cardiomyopathy and ventricuamiodarone in patients with hypertrophic

17 Fananapazir L, Chang A, Epstein S, McAreavey D. Prognostic determinants in hypertrophic cardiomyopathy. Prospective evaluation of a nants in hypertrophic cardiomyopathy. Prospective evaluation of a therapeutic strategy based on clinical, Holter, hemo

18 Saumarez RC, Camm AJ, Panagos A, Gill JS, et al. Ventricular fibrillation in hypertrophic cardiomyopathy is associated with increased fractionain hypertrophic cardiomyopathy is associated with increased fractiona-
tion of paced right ventricular electrograms. Circulation 1992;86:467-74.

19 McKenna WJ, Camm AJ. Editorial Comment: Sudden death in hyperMcKenna WJ, Camm AJ. Editorial Comment: Sudden death in hyper-
trophic cardiomyopathy: assessment of patients at high risk. Circulation

20 Clark A, Coats A. Screening for hypertrophic cardiomyopathy. BMF 1993;306:409-10.

\section{COMMENTARY}

\section{Are polymorphisms in the ACE gene a potent genetic risk factor for restenosis?}

The three forms of the gene for the human angiotensin converting enzyme (ACE) are determined by the insertion (I) or deletion (D) of an extra sequence in the DNA code. These forms are DD (two deletions), ID (one deletion), and II (no deletion). People who inherit the DD form of the gene have higher concentrations of circulating $\mathrm{ACE}$ than those who have one or two insertions. ${ }^{1}$ Because ACE, through the activation of angiotensin II, is a potent stimulator of vascular smooth muscle proliferation it is possible that those who inherit the DD form of the gene are at a higher risk of restenosis after angioplasty. A recent study by Ohishi et al suggests that this may be so. ${ }^{2}$

Eighty two consecutive patients with myocardial infarction who had successful emergency coronary angioplasty within 24 hours of the onset of infarction were studied. All had follow up angiography 3-6 months later. Restenosis was defined as a lesion producing more than a $50 \%$ reduction in lumen diameter at the angioplasty site. A method based on the polymerase chain reaction was used to detect the ACE gene polymorphism in DNA extracted from peripheral leucocytes. The DD genotype was found in 37 (45\%) patients compared with $16(15 \%)$ of 102 controls. A higher frequency of the DD genotype in those with acute infarction has been reported by others. ${ }^{3}$ The results of the restenosis study, which are summarised in the table, show that compared with the ID and II genotypes, the DD genotype was more frequent in patients who had restenosis than in patients without restenosis. The odds ratio, an estimate of relative risk of restenosis between patients with the DD genotype and those with ID or II genotypes, was $4: 1$. Thus the study suggests that the DD genotype of ACE is a potent risk factor for restenosis after emergency angioplasty. ${ }^{2}$

Comparison of restenosis rate in patients with different $A C E$ genotypes

\begin{tabular}{lll}
\hline & Genotype \\
\cline { 2 - 3 } & $D D(\%)$ & ID or II (\%) \\
\hline Restenosis & $21(56 \cdot 8)$ & $11(24 \cdot 4)$ \\
No restenosis & $16(43 \cdot 2)$ & $34(75 \cdot 6)$ \\
Total & $37(100)$ & $45(100)$ \\
\hline
\end{tabular}

$\chi^{2}=8.91(P=0.0028)$
The implications of the study, ${ }^{2}$ if the results are confirmed in a larger series of patients and different geographic populations, are important. First, it may be necessary to characterise the ACE genotype in patients undergoing angioplasty to identify those patients who are at higher risk of restenosis. Treatment with ACE inhibitors may benefit this group. In addition the numerous studies that have assessed the role of different risk factors for restenosis after coronary angioplasty may need re-evaluation. The results of large trials, such as the MERCATOR study, ${ }^{4}$ designed to test the hypothesis that ACE inhibition may prevent restenosis in patients after balloon angioplasty, may require re-analysis.

Certain factors in the study reported by Ohishi et al may have influenced the results. The angiographic analysis was by visual means alone, the definition of restenosis arbitrary, and the study group small and selected. Moreover, because only Japanese patients were studied, it is possible that ethnic differences could have had a role. In addition, though the frequency of the DD genotype was significantly higher in patients with restenosis, many patients without restenosis also had the DD genotype.

JUAN CARLOS KASKI

Coronary Artery Disease Research Group,

Department of Cardiological Sciences,

St George's Hospital Medical School,

London

Rigat B, Hubert C, Alhenc-Gelas F, Cambien F, Corvol P, Soubrier F An insertion/deletion polymorphism in the angiotensin 1-converting enzyme gene accounting for half the variance of serum enzyme levels. $\not{f}$ Clin Invest 1990;86:1343-6.

2 Ohishi M, Fujii K, Minamino T, et al. A potent genetic risk factor for restenosis. Nature Genetics 1993;5:324-5.

3 Cambien F, Poirier O, Lacerf L, et al. Deletion polymorphism in the gene for angiotensin-converting enzyme is a potent risk factor for myocardial infarction. Nature 1992;359:641-4.

4 Mercator Study Group. Does the new angiotensin converting enzyme inhibitor cilazapril prevent restenosis after percutaneous transluminal coronary angioplasty? Results of the MERCATOR study: a multicencoronary angioplasty? Results of the MERCATOR study: a multicentre, randomized, double blind placebo-controlled trial. Mulcentre European Research Trial with cilazapril after angioplasty to prevent 1992;86:100-10. 